\title{
Altered spontaneous brain activity patterns in strabismus with amblyopia patients using amplitude of low-frequency fluctuation: a resting-state $\mathrm{fMRI}$ study
}

This article was published in the following Dove Press journal:

Neuropsychiatric Disease and Treatment

\author{
You-Lan Min ${ }^{1, *}$ \\ Ting $\mathrm{Su}^{2, *}$ \\ Yong-Qiang Shu ${ }^{3}$ \\ Wen-Feng Liu' \\ Ling-Long Chen ${ }^{3}$ \\ Wen-Qing Shi' \\ Nan Jiang ${ }^{2}$ \\ Pei-Wen Zhu' \\ Qing Yuan' \\ Xiao-Wei Xu' \\ Lei Ye' \\ Yi Shao'
}

'Department of Ophthalmology, The First Affiliated Hospital of Nanchang University, Jiangxi Province Clinical Ophthalmology Institute, Nanchang 330006 Jiangxi, China; ${ }^{2}$ Eye Institute of Xiamen University, Fujian Provincial Key Laboratory of Ophthalmology and Visual Science, Xiamen 36II02, Fujian, China; ${ }^{3}$ Department of Radiology, The First Affiliated Hospital of Nanchang University, Nanchang 330006 Jiangxi, China

*These authors contributed equally to this work

Correspondence: Yi Shao

Department of Ophthalmology, The First Affiliated Hospital of Nanchang University, Jiangxi Province Clinical Ophthalmology Institute, 17 Yongwaizheng Street, Donghu, Nanchang, Jiangxi 330006, China Email freebee99@।63.com
Objective: Previous studies have demonstrated that strabismus or amblyopia can result in markedly brain function and anatomical alterations. However, the differences in spontaneous brain activities of strabismus with amblyopia (SA) patients still remain unclear. This current study intended to use the amplitude of low-frequency fluctuation (ALFF) technique to investigate the intrinsic brain activity changes in SA subjects.

Patients and methods: A total of 16 patients with SA ( 6 males and 10 females) and 16 healthy controls (HCs; 6 males and 10 females) similarly matched in age, gender, and education status were recruited and examined with the resting-state functional MRI. The spontaneous brain activity changes were investigated using the ALFF technique. The receiver operating characteristic curve was performed to classify the mean ALFF signal values of the SA patients from HCs. The correlations between the ALFF values of distinct brain regions and the clinical manifestations in SA patients were evaluated in terms of the Pearson's correlation analysis.

Results: Compared with HCs, SA patients had significantly decreased ALFF in the left cerebellum posterior lobe, left middle frontal gyrus, and bilateral thalamus. In contrast, SA patients showed increased ALFF values in the right superior frontal gyrus, right precuneus, left cuneus, and bilateral precentral gyrus. Nonetheless, there was no linear correlation between the mean ALFF values in brain regions and clinical features.

Conclusion: Diverse brain regions including vision-related and motion-related areas exhibited aberrant intrinsic brain activity patterns, which imply the neuropathologic mechanisms of oculomotor disorder and vision deficit in the SA patients.

Keywords: strabismus, amblyopia, functional MRI, ALFF, spontaneous brain activity

\section{Introduction}

Strabismus and amblyopia are two common visual development disorders, which might have onset in infancy but persist into adulthood if not successfully treated. ${ }^{1,2}$ Strabismus is an optical manifestation disorder in coordination of the extraocular muscles, which is considered to be related to the cerebral visual pathways maldevelopment that mediate eye movements, ${ }^{3}$ and inimically affecting the stereopsis, binocularity, and depth of perception. ${ }^{4}$ It has been reported that the incidence rate of adult-onset strabismus was 54.2 per 100,000 individuals, ${ }^{5}$ and adult strabismus is usually associated with amblyopia. ${ }^{6}$ Amblyopia is a monocular visual disorder owing to abnormal binocular development, and generally identified by decreased visual acuity (VA) and sensitivity in the amblyopic eye. ${ }^{7}$ Strabismus often contributes to compromised amblyopia, and 
amblyopia might lead to perceptual strabismus. In addition to the functional effects of strabismus and amblyopia, there are often esthetic concerns that can subsequently affect daily psychosocial performance..$^{8-10}$

MRI has progressed rapidly in recent years, providing a noninvasive nerve imaging method to evaluate the functional and structural alterations in the human brains. ${ }^{11}$ And functional MRI (fMRI) is a commonly used brain function detection technique, which has been considered to have an accurate spatial resolution. ${ }^{12}$ Based on cerebral blood flow and metabolism analysis, researchers can detect specific brain region activation to explore the spatial organization of the brain, including the visual pathway (from retina to cortex), and assist to reveal the mechanisms of the eye disease. ${ }^{13-15}$

Some researchers have used fMRI techniques to show brain changes in patients with strabismus or amblyopia, respectively. Previous studies revealed altered regional cerebral blood flow and blood oxygen level-dependent (BOLD) signal in visual cortex, ${ }^{16,17}$ extrastriate cortex, ${ }^{18}$ and lateral geniculate nucleus of amblyopia. ${ }^{19,20}$ Furthermore, studies on diverse types of strabismus also exhibited abnormal activity in specific regions of the brain both under tasking state and resting state. ${ }^{21-23}$ However, previous studies have only focused on the strabismus or amblyopia, respectively.

Amplitude of low-frequency fluctuation (ALFF) is a resting-state fMRI analysis method, which is able to manifest regional spontaneous brain activities at rest by evaluating the intrinsic fluctuations in BOLD signal. This present study proposed to use the ALFF technique to explore altered spontaneous brain activities in strabismus with amblyopia (SA) patients in comparison with healthy controls (HCs) and correlate the results with clinical manifestations.

\section{Patients and methods}

\section{Subjects}

A total of 16 patients with SA (6 males and 10 females, 11 exotropia and 5 esotropia) were recruited from the Ophthalmology Department of the First Affiliated Hospital of Nanchang University. Inclusion criteria: (1) adults over 18 years old; (2) strabismus; (3) there had to be greater than a one line difference in the best-corrected VA (VA $\geq 0.20$ logMAR units) between the amblyopic and the fellow eye, and had central fixation; (4) the eyes did not combine with other eye diseases (cataract, glaucoma, optic neuritis, macular degeneration, etc.). Exclusion criteria: (1) patients with previous ocular surgery history (intraocular surgery and extraocular surgery); (2) any evidence of other eye disease (infection, inflammation, and ischemic disease); (3) psychiatric disorders, cardiovascular disease, and cerebral infarction disease; (4) drugs or alcohol addiction.

Sixteen HCs ( 6 males and 10 females) with similar age, gender, and education status compared to the SA group participated in this study. All of the HCs met the subsequent criteria: (1) no deformities in the brain parenchyma on MRI; (2) no ocular disease history with best-corrected VA $\leq 0$ logMAR units; (3) no psychiatric disease; (4) capable of MRI examination (no cardiac pacemaker or implanted metal devices, etc.).

This study was approved by the Medical Ethics Committee of the First Affiliated Hospital of Nanchang University and followed the Declaration of Helsinki. All subjects cooperated voluntarily and signed informed consents after informing of the purposes, contents, and potential risks.

\section{MRI parameters}

MRI scans were performed on 3-Tesla MRI scanners (Trio, Siemens, Munich, Germany). All participants were asked to keep their eyes closed, stay awake, and breath calmly until the scan was over. Then the functional data were collected using a 3D spoiled gradient-recalled echo sequence with the following parameters: 176 structural images (repetition time $=1,900 \mathrm{~ms}$, echo time $=2.26 \mathrm{~ms}$, thickness $=1.0 \mathrm{~mm}$, gap $=0.5 \mathrm{~mm}$, acquisition matrix $=256 \times 256$, field of view $=$ $250 \times 250 \mathrm{~mm}$, flip angle $\left.=9^{\circ}\right)$. And 240 functional images (repetition time $=2,000 \mathrm{~ms}$, echo time $=30 \mathrm{~ms}$, thickness $=$ $4.0 \mathrm{~mm}$, gap $=1.2 \mathrm{~mm}$, acquisition matrix $=64 \times 64$, flip angle $=90^{\circ}$, field of view $=220 \times 220 \mathrm{~mm}, 29$ axial) were also obtained. The whole scanning time was $15 \mathrm{~min}$.

\section{fMRI data processing}

We implied MRIcro software to classify functional data to exclude incomplete data. The first 15 time points were discarded owing to magnetization equilibration. The rest of the data preprocessing, such as Digital Imaging Communications in Medicine form transformation, slice timing, head motion correction, spatial normalization, and smoothening with a Gaussian kernel of $6 \times 6 \times 6 \mathrm{~mm}^{3}$ full-width at half-maximum, was performed by the toolbox Data Processing Assistant for Resting-State fMRI advanced edition (DPARSFA 4.0, http://rfmri.org/DPARSF) software based on Statistical Parametric Mapping software (SPM, http://www.fil.ion.ucl.ac.uk/spm) and a rs-fMRI data analysis toolkit (REST, http://www.restfmri.net) software. The subjects who had more than $1.5 \mathrm{~mm}$ maximum shift in $\mathrm{x}, \mathrm{y}$, or $\mathrm{z}$ and 1.5 of angular motion during the entire 
fMRI scan were eliminated. The Friston six head motion parameters were used to regress out head motion effects based on recent work showing that higher-order models were more effective in removing head-motion effects. ${ }^{24}$ Linear regression was also implied to remove false variables from other sources, which contain areas of ventricular region of interest (ROI) and a region centered in the brain white matter. After the correction of the head motion, the standard echoplanar image template was used to standardize the functional images in reaching the Montreal Neurological Institute (MNI) space criteria. The ALFF of each voxel was divided by the global mean ALFF value for each participant to reduce the global effects of variability.

\section{Brain-behavior correlation analysis}

Brain regions with various ALFF values between the two groups were classified as ROI using the REST software, and the mean ALFF of each ROI was calculated by averaging every original ALFF value over all voxels. In the SA group, the relationship between the mean ALFF value in each ROI and behavioral performances was investigated with linear correlation analysis. $P<0.05$ was considered to be statistically significant.

\section{Statistical analysis}

The demographic and clinical variables between SA and HC groups were compared using SPSS20.0 software (SPSS, IBM Corporation, Armonk, NY, USA) with independent sample $t$-test, and the results were considered to be statistically significant when $P<0.05$.

Two-sample $t$-test was applied to explore the voxel-wise difference between two groups using the REST software. The statistical threshold of voxel level was set at $P<0.05$ for multiple comparisons using Gaussian random field theory. And AlphaSim corrected at a voxel level of $P<0.01$ and cluster size $>40$ voxels.

The receiver operating characteristic (ROC) curve method was performed to classify the mean ALFF values in diverse brain regions of the SA subjects from HCs. The correlations between the ALFF values of distinct brain areas and the clinical features in SA patients were evaluated in terms of the Pearson's correlation analysis.

\section{Results}

\section{Demographics and visual measurements}

There were no significant differences in age $(P=0.615)$ and best-corrected VA of fellow eye $(P=0.185)$ between the
Table I Demographics and clinical measurements of SA and HC groups

\begin{tabular}{lllll}
\hline & SA & HC & t-value & P-value \\
\hline Male/female & $6 / 10$ & $6 / 10$ & $\mathrm{~N} / \mathrm{A}$ & $>0.99$ \\
Age (years) & $24.50 \pm 5.9 \mathrm{I}$ & $24.94 \pm 5.23$ & -0.222 & 0.615 \\
Handedness & $16 \mathrm{R}$ & $\mathrm{I} 6 \mathrm{R}$ & $\mathrm{N} / \mathrm{A}$ & $>0.99$ \\
Duration (years) & $18.19 \pm 9.85$ & $\mathrm{~N} / \mathrm{A}$ & $\mathrm{N} / \mathrm{A}$ & $\mathrm{N} / \mathrm{A}$ \\
Esotropia/exotropia & $5 / \mathrm{II}$ & $\mathrm{N} / \mathrm{A}$ & $\mathrm{N} / \mathrm{A}$ & $\mathrm{N} / \mathrm{A}$ \\
Spherical equivalent & $\mathrm{I} .22 \pm 0.56$ & $\mathrm{I} .25 \pm 0.67$ & -0.365 & $0.74 \mathrm{I}$ \\
refractive error (diopters) & & & & \\
Mean \pm SD (range) & $-2.75-1.75$ & $-2.75-2.00$ & $\mathrm{~N} / \mathrm{A}$ & $\mathrm{N} / \mathrm{A}$ \\
Angle of strabismus (PD) & $26.25 \pm 12.7 \mathrm{I}$ & $\mathrm{N} / \mathrm{A}$ & $\mathrm{N} / \mathrm{A}$ & $\mathrm{N} / \mathrm{A}$ \\
Best-corrected VA-AE & 0.53 & $-0.05 \pm 0.08$ & 6.149 & $<0.00 \mathrm{I}$ \\
Best-corrected VA-FE & $-0.03 \pm 0.09$ & $-0.0 \mathrm{I} \pm 0.07$ & $-0.65 \mathrm{I}$ & 0.185 \\
\hline
\end{tabular}

Notes: Independent $t$-tests comparing the two groups $(P<0.05$ represented statistically significant differences). Data shown as mean SD or $n$.

Abbreviations: $\mathrm{AE}$, amblyopic eye; $\mathrm{FE}$, fellow eye; $\mathrm{HC}$, healthy control; N/A, not applicable; PD, prism diopter; R, right; SA, strabismus with amblyopia; VA, visual acuity.

SA patients and the HCs. There were statistically notable differences in the best-corrected VA of amblyopic eye $(P<0.001)$ between the two groups (more details are presented in Table 1).

\section{ALFF differences}

In the SA group, the ALFF values were significantly increased in the right superior frontal gyrus and precuneus, left cuneus, and bilateral precentral gyrus compared to $\mathrm{HCs}$ (Figure 1 [red] and Table 2). The brain regions of the SA group with significantly decreased ALFF values included the bilateral thalamus, left cerebellum posterior lobe, and middle frontal gyrus (Figure 1 [blue] and Table 2). The mean values of ALFF between two groups are presented in Figure 2. However, we did not discover any correlation between the ALFF values of different brain regions and their manifestations in the SA group $(P>0.05)$.

\section{ROC curve}

We hypothesized that the differences in ALFF values could be potential diagnostic markers to distinguish the SA group from HCs. To verify this assumption, the mean ALFF values of the distinct brain areas in SA group were collected and analyzed using ROC curves. When the area under the curve (AUC) is 0.5-0.7, it denotes the accuracy is low, and the accuracy is certain when AUC is 0.7-0.9. The individual AUC of ALFF values in different regions were as follows: right superior frontal gyrus $(0.871, P<0.001)$, right precuneus (0.949, $P<0.001)$, left cuneus $(0.898, P<0.001)$, bilateral precentral gyrus $(0.957, P<0.001)$, (Figure $3 \mathrm{~A}, \mathrm{SAs}>\mathrm{HCs})$, 
A

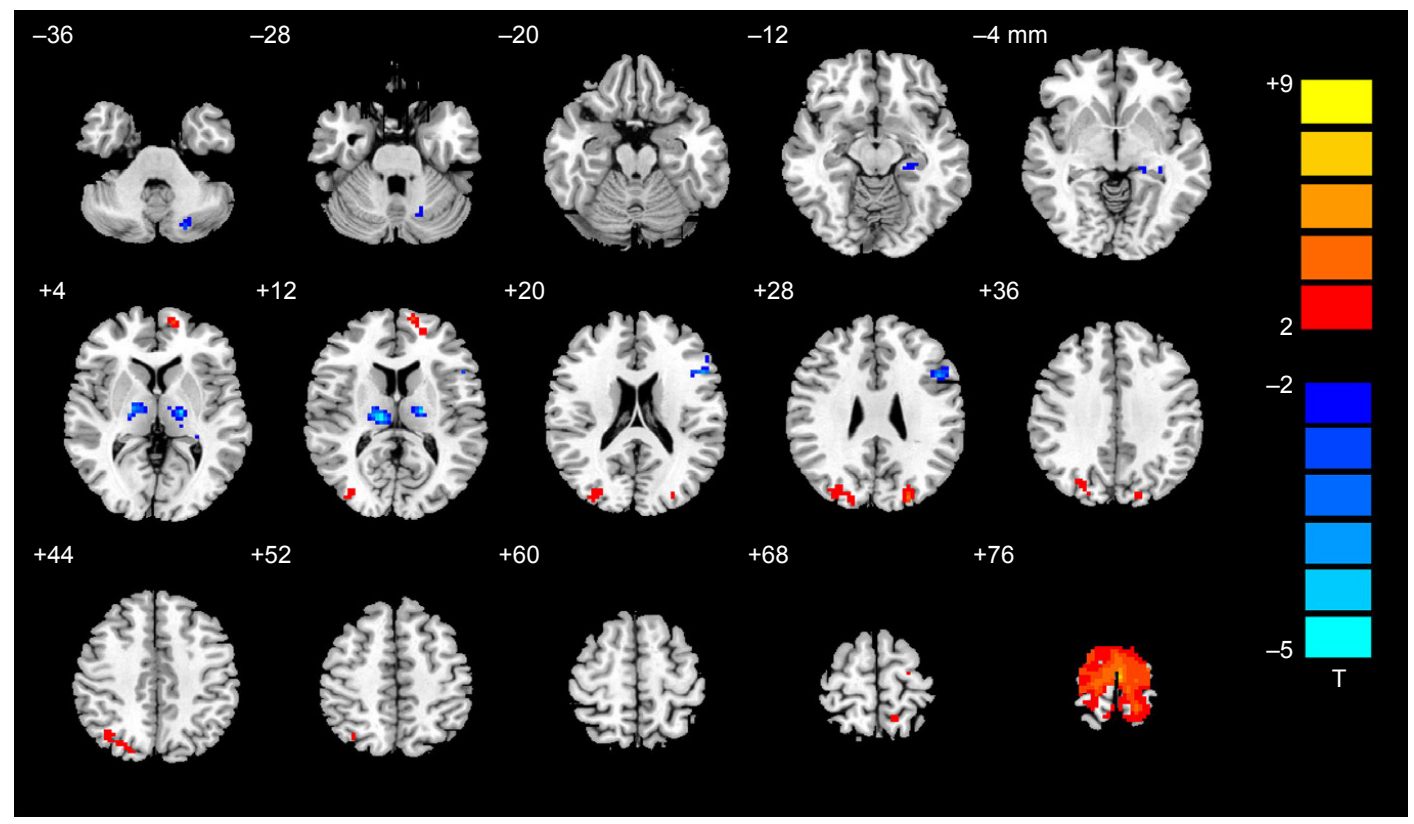

B L
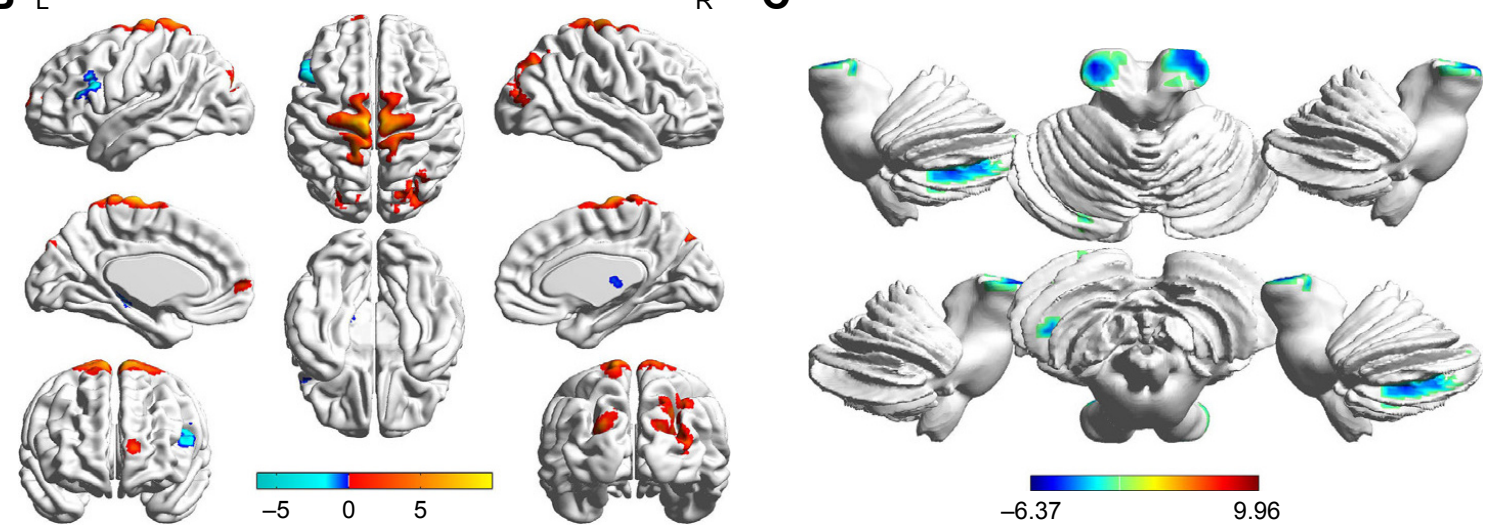

Figure I Spontaneous brain activity in the SA and HC groups.

Notes: (A) The different ALFF regions between the SA and HC groups. (B) Significant differences of brain activity in cerebrum. (C) Significant differences of brain activity in cerebellum. The red regions indicate higher ALFF values, and the blue regions imply lower ALFF values $(P<0.05$, AlphaSim corrected, cluster size $>40)$.

Abbreviations: ALFF, amplitude of low-frequency fluctuation; HC, healthy control; L, left; R, right; SA, strabismus with amblyopia.

Table 2 Brain areas with significantly different ALFF values between groups

\begin{tabular}{|c|c|c|c|c|c|c|c|c|}
\hline \multirow[t]{2}{*}{ Conditions } & \multirow[t]{2}{*}{ L/R } & \multirow[t]{2}{*}{ Brain regions } & \multirow[t]{2}{*}{ BA } & \multicolumn{3}{|c|}{ MNI coordinates } & \multirow{2}{*}{$\begin{array}{l}\text { Peak } \\
\text { voxels }\end{array}$} & \multirow[t]{2}{*}{$t$-value } \\
\hline & & & & $x$ & $Y$ & $\mathbf{Z}$ & & \\
\hline \multicolumn{9}{|l|}{$\mathrm{SAs}>\mathrm{HCs}$} \\
\hline 1 & $\mathrm{R}$ & Superior frontal gyrus & 10 & -12 & 60 & 6 & 41 & 4.6552 \\
\hline 2 & $\mathrm{R}$ & Precuneus & & 27 & -75 & 39 & 144 & 4.8382 \\
\hline 3 & $\mathrm{~L}$ & Cuneus & 19 & -24 & -87 & 27 & 48 & 5.1766 \\
\hline 4 & B & Precentral gyrus & 6 & -3 & -21 & 78 & 648 & 9.9577 \\
\hline \multicolumn{9}{|l|}{$\mathrm{SAs}<\mathrm{HCs}$} \\
\hline 1 & $\mathrm{~L}$ & Cerebellum posterior lobe & & -33 & -60 & -42 & 88 & -4.4430 \\
\hline 2 & $\mathrm{~L}$ & Thalamus & & -18 & -15 & 12 & 78 & -6.3723 \\
\hline 3 & $\mathrm{R}$ & Thalamus & & 12 & -21 & 12 & 101 & -6.0901 \\
\hline 4 & $\mathrm{~L}$ & Middle frontal gyrus & 9 & -48 & 15 & 30 & 89 & -5.6140 \\
\hline
\end{tabular}

Note: The statistical threshold was set at voxel level with $P<0.05$ for multiple comparisons using Gaussian random field theory voxels with $P<0.01$ and cluster size $>40$ voxels, AlphaSim corrected.

Abbreviations: ALFF, amplitude of low-frequency fluctuation; BA, Brodmann area; B, bilateral; HC, healthy control; L, left; MNI, Montreal Neurological Institute; R, right; SA, strabismus with amblyopia. 


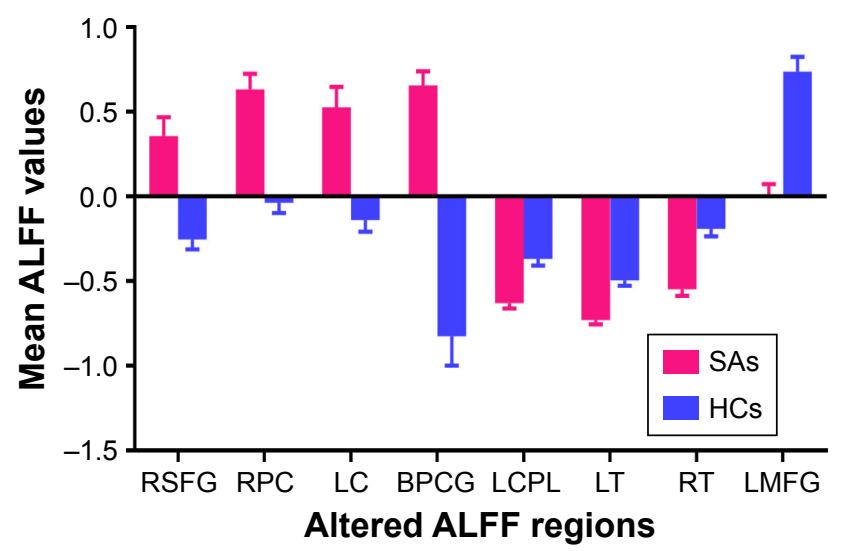

Figure 2 The mean ALFF values between the SA and HC groups. Abbreviations: ALFF, amplitude of low-frequency fluctuation; BPCG, bilateral precentral gyrus; HC, healthy control; LC, left cuneus; LCPL, left cerebellum posterior lobe; LMFG, left middle frontal gyrus; LT, left thalamus; RPC, right precuneus; RSFG, right superior frontal gyrus; RT, right thalamus; SA, strabismus with amblyopia.

left cerebellum posterior lobe $(0.914, P<0.001)$, left thalamus (0.938, $P<0.001)$, right thalamus $(0.938, P<0.001)$, and left middle frontal gyrus $(0.973, P<0.001)$. (Figure $3 \mathrm{~B}$, SAs $<\mathrm{HCs}$ ).

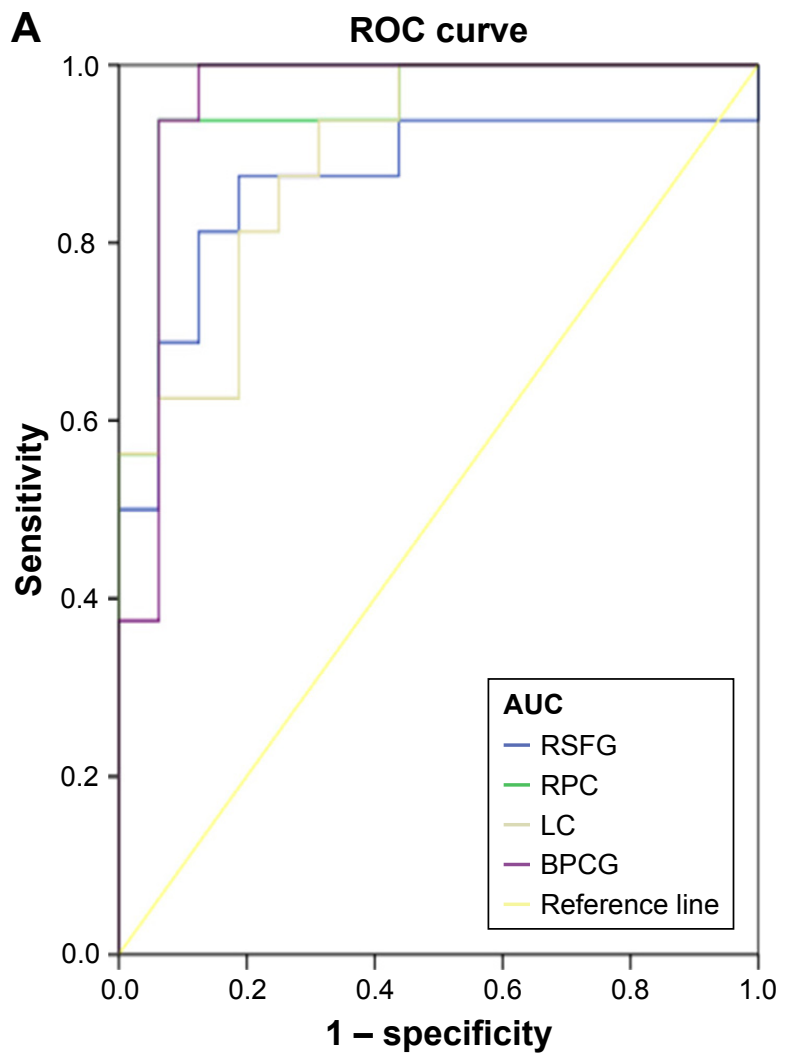

\section{Discussion}

To the best of our knowledge, this is the very first study to demonstrate that the intrinsic brain activity patterns of different regions in the SA individuals were altered when compared to HCs.

\section{Analysis of higher ALFF values in the SA group}

The SA patients had significantly increased ALFF signal values in the left cuneus, right precuneus, right superior frontal gyrus, and bilateral precentral gyrus.

The cuneus, located in the occipital lobe which involves the visual cortex, is believed to play an important role in visual processing. ${ }^{25}$ It has been reported that the monocular inputs are originally combined in primary visual cortex (V1), where alteration might relate to amblyopic vision deficit. ${ }^{26}$ In addition, the cuneus modifies visual information to extrastriate cortices via V1. ${ }^{27}$ Previous studies have shown inconsistency in this region. Some claimed to observe reduced cerebral blood flow in V1 and extrastriate

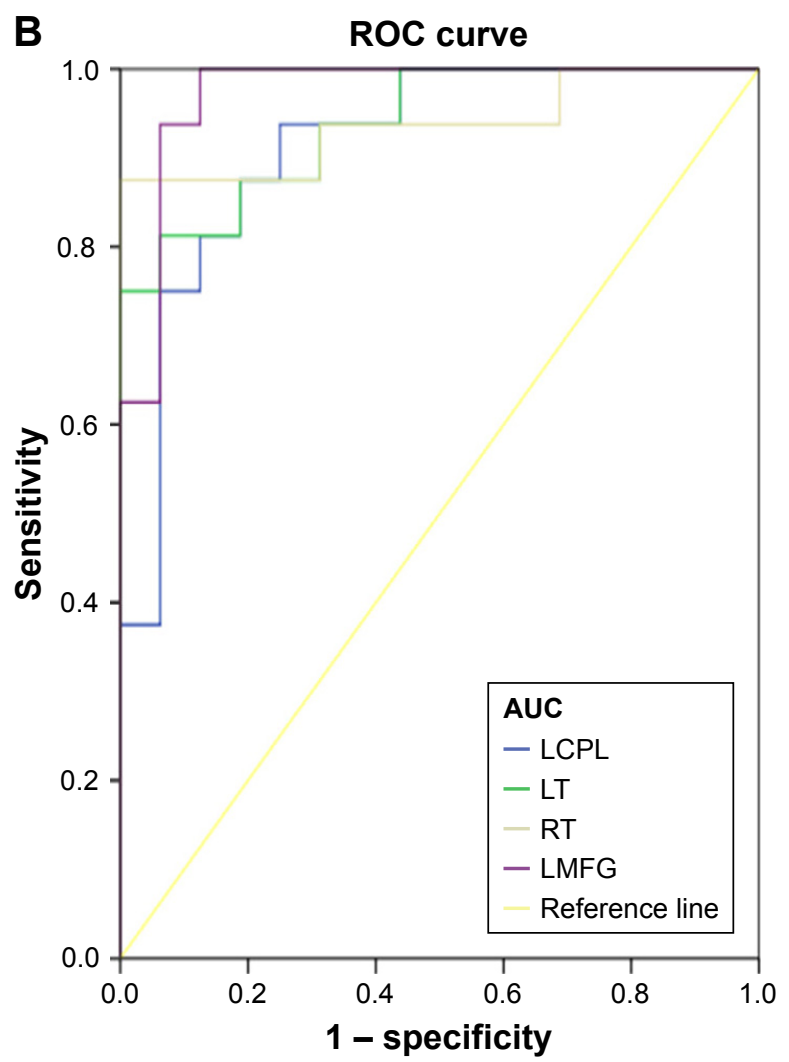

Figure 3 ROC curve analysis of the mean ALFF values for altered brain regions in SA group.

Notes: $(\mathbf{A})$ The area under the ROC curve were 0.87I, $(P<0.00$ I; $95 \%$ Cl: $0.734-1.000)$ for RSFG, RPC 0.949 ( $P<0.00$ I; $95 \%$ Cl: $0.874-I .000)$, LC 0.898 ( $P<0.00$ I; $95 \%$ Cl: 0.795-I.000), BPCG 0.957 ( $P<0.00$ I; $95 \%$ Cl: 0.879-I.000) (SAs $>$ HCs). (B) The area under the ROC curve were 0.914 ( $P<0.00$ I; $95 \%$ Cl: $0.815-1.000)$ for LCPL, LT 0.938 $(P<0.00$ I; $95 \%$ Cl: 0.859-1.000), RT 0.938 ( $P<0.001$; 95\% Cl: 0.845-I.000), LMFG 0.973 ( $P<0.001$; $95 \%$ Cl: $0.922-1.000)(S A s<$ HCs).

Abbreviations: ALFF, amplitude of low-frequency fluctuation; AUC, area under the curve; BPCG, bilateral precentral gyrus; HC, healthy control; LC, left cuneus; LCPL, left cerebellum posterior lobe; LMFG, left middle frontal gyrus; LT, left thalamus; ROC, receiver operating characteristic; RPC, right precuneus; RSFG, right superior frontal gyrus; RT, right thalamus; SA, strabismus with amblyopia. 
cortex of amblyopic eye during visual stimulation, ${ }^{16,28,29}$ and reduced gray matter volume in the visual cortex included bilaterally cuneus in adults with strabismus. ${ }^{30}$ Meanwhile, Li et $\mathrm{al}^{31}$ have found white matter volume increased in the right cuneus, middle occipital, and left orbitofrontal regions of monocular amblyopia patients under resting state. In this study, the SA group showed higher ALFF values in the left cuneus. Such increase is supposed to imply the plasticity of neurons, probably driven by stimulation from the fellow eye, afterward leading to a compensatory increase for the deficit of visual input.

The precuneus is located in the medial wall of BA7, which is the somatosensory association cortex and believed to play a pivotal role in visuomotor coordination. Precuneus has been suggested to be involved in visuospatial imagery, ${ }^{32}$ selfprocessing, ${ }^{33}$ episodic memory retrieval, ${ }^{34}$ spatial location encoding, ${ }^{35}$ and default-mode network. ${ }^{36}$ In previous studies, Wang $^{37}$ and Muckli ${ }^{38}$ have demonstrated that visuomotor coordination is decreased in amblyopic subjects. Similar results have displayed declined ALFF in precuneus both in amblyopic children and adults..$^{39,40}$ Researches of strabismus subjects suggested that white matter volume was reduced in the right precuneus in the comitant strabismus patients. ${ }^{41}$ Nevertheless, Yang et al found a significant increase of the bold signal in the bilateral precuneus in the patients with infantile esotropia, ${ }^{42}$ and cortical activations in intermittent exotropia were found in right precuneus. ${ }^{23}$ In the present study, we detected an increased ALFF in the right precuneus of SA group. This escalation is consistent with the cuneus, which suggest an analogous potential compensation mechanism may facilitate visual defective individuals to execute sensory-guided motor behaviors.

The precentral gyrus is located on the superficial posterior frontal lobe, which is a part of the primary motor cortex that contains multiple neurons and associates with the muscle. ${ }^{43}$ The precentral gyrus plays a critical part in movement frequency and quantity. ${ }^{44,45}$ Greater gray matter volume was identified at the right precentral gyrus in subjects with strabismus. ${ }^{30}$ Lin et $\mathrm{al}^{46}$ reported that the regional homogeneity values of spontaneous brain activity in the bilateral precentral and postcentral gyrus was higher in anisometropic amblyopia. Consistent with these previous findings, the increased ALFF value in our study may reflect the plasticity that compensates for strabismus and amblyopiarelated deficits.

In the oculomotor pathway of saccadic eye movement, some brain regions are thought to be implicated, such as the frontal eye field (FEF), the supplementary eye field (SEF), and midbrain regions. ${ }^{47}$ The SEF is located on the superior section of the frontal lobe medial wall, ${ }^{48}$ where superior frontal gyrus lies in, which indirectly controls the sequences of visual-guided saccades and eye-hand coordination. Studies have detected greater gray matter volume at the left superior frontal gyrus and inferior frontal sulcus in patients with strabismus. ${ }^{30}$ In this current study, higher ALFF value was observed in the right superior frontal gyrus of SA group in comparison with HCs. These findings indicated functional reorganization to offset the neighboring impaired brain areas.

\section{Analysis of lower ALFF values in the SA group}

The SA patients had significantly decreased ALFF signal values in the left middle frontal gyrus, cerebellum posterior lobe, and bilateral thalamus.

The FEF is located in the frontal cortex, which is capable of initiating eye movements and influencing their latency or accuracy. ${ }^{49}$ The restricted FEF location is believed to lie on the posterior area of the middle frontal gyrus within a larger oculomotor region. ${ }^{50}$ Previous studies illustrated that the FEF played a crucial role in saccade related to movement generation. ${ }^{51,52}$ The patients with comitant exotropia exhibited decreased white matter volumes in the frontal lobe of the right hemisphere, ${ }^{53}$ meanwhile the amblyopic subjects displayed reduced gray matter density in the left middle frontal gyrus. ${ }^{39}$ In addition, voxel-wise degree centrality (DC) values of comitant exotropia strabismus patients decreased in the right middle frontal gyrus. ${ }^{54}$ Furthermore, Tan et $\mathrm{al}^{22}$ reported lower ALFF values of the bilateral middle frontal gyrus in subjects with congenital comitant strabismus, and similar ALFF reduction was found in anisometropic amblyopia patients. ${ }^{55}$ In support of these preceding reports, we also inspected decreased ALFF values in the left middle frontal gyrus in the SA group, which indicated FEF functional injury in these patients.

The cerebellum posterior lobe is located beneath the primary fissure and plays an important role in motor control and perception, ${ }^{56}$ particularly including the control of ocular motor. ${ }^{57-59}$ A previous study have provided evidence that the cerebellum was associated with the execution of eye movements. ${ }^{60}$ Hayakawa et al ${ }^{58}$ suggested that the cerebellar posterior vermis was related to saccadic eye movements. Huang et $\mathrm{al}^{61}$ found the mean diffusivity values in the bilateral cerebellum posterior lobe were significantly decreased in comitant strabismus patients, and Tan et $\mathrm{al}^{54}$ found DC values of comitant exotropia strabismus patients reduced 
in the right cerebellum posterior lobe. Moreover, lower ALFF value of strabismus subjects has been observed in the bilateral cerebellum posterior lobe. ${ }^{22}$ In line with these previous studies, the ALFF reduction in the left cerebellum posterior lobe of SA group shown in the current study may reflect functional damage in this area. Therefore, we further presumed that this might result in the motor control impairment in SA subjects.

The thalamus is a vital region integrating neural activities from widespread cortical inputs and outputs. ${ }^{62}$ The thalamus controls the information transmission to cortex and is involved in visual perception and dynamic visual information processing to motor center via the retino-thalamocortical pathways. ${ }^{63}$ A previous study demonstrated that greater gray matter volume was detected at the right side of the thalamus of strabismus adults. ${ }^{30}$ Gupta et $a l^{64}$ found that viewing with amblyopic eye exhibited BOLD activity in bilateral thalamus with respect to amblyopia, whereas control group presented greater BOLD neural activation in cerebellum, thalamus, and frontal cortex compared with SA patients. In the present study, the ALFF reduction provided new evidence that the SA may lead to dysfunction of the thalamus.

Previous studies about amblyopia patients revealed altered ALFF values of brain regions in calcarine, middle occipital gyrus, postcentral gyrus, and precuneus, which were different from our current results. This might indicate the patients with strabismus would lead to brain functional impairment in superior frontal gyrus, cuneus, precentral gyrus, middle frontal gyrus, cerebellum posterior lobe, and thalamus. Studies about strabismus patients reported altered ALFF values of brain regions in medial frontal gyrus, cerebellum posterior lobe, and angular gyrus, which were clearly differ from the present study. This could help to explain the potential injury in superior frontal gyrus, precuneus, cuneus, precentral gyrus, and thalamus of patients with amblyopia. This study illustrated the different altered brain regions in SA patients and provided basis for further exploration of the potential pathogenesis of SA.

\section{Conclusion}

In summary, our results demonstrated that patients with SA had abnormal spontaneous activities in specific brain regions, which provide insight into the neural variation in SA patients.

However, several limitations have existed in the current study include a small number of sample size, which should be expanded in the future research for more precise results.
And the clinical characteristics were not rigorous, for instance, exotropia and esotropia were all implicated. Different types of strabismus and amblyopia should be distinguished in the future research so as to evaluate brain functional activity changes more accurately. In spite of these defects, the present study revealed the potential pathogenesis of SA was relevant to the impairment in specific brain regions.

\section{Acknowledgments}

This study was supported by the National Natural Science Foundation of China (No: 81660158, 81460092, 81400372), Jiangxi Province Voyage Project (No: 2014022), Natural Science Key Project of Jiangxi Province (No: 20161ACB21017, 20181BBG70004), Youth Science Foundation of Jiangxi Province (No: 20151BAB215016), Technology and Science Foundation of Jiangxi Province (No: 20151BBG70223), and Health Development Planning Commission Science Foundation of Jiangxi Province (No: 20155204, 20175116).

\section{Disclosure}

This was not an industry supported study. The authors report no conflicts of interest in this work.

\section{References}

1. Chen X, Fu Z, Yu J, et al. Prevalence of amblyopia and strabismus in Eastern China: results from screening of preschool children aged 36-72 months. Br J Ophthalmol. 2014;100(4):515-519.

2. Tarczy-Hornoch K, Cotter SA, Borchert M, et al. Prevalence and causes of visual impairment in Asian and non-Hispanic white preschool children: multi-ethnic pediatric eye disease study. Ophthalmology. 2013;120(6):1220-1226.

3. Brodsky MC, Fray KJ, Glasier CM. Perinatal cortical and subcortical visual loss: mechanisms of injury and associated ophthalmologic signs. Ophthalmology. 2002;109(1):85-94.

4. Gunton KB, Wasserman BN, Debenedictis C. Strabismus. Prim Care. 2015;42(3):393-407.

5. Mohney BG, Martinez JM, Holmes JM, Diehl NN. Incidence of strabismus in an adult population. J AAPOS. 2012;16(1):e23.

6. Chan ST, Tang KW, Lam KC, Chan LK, Mendola JD, Kwong KK. Neuroanatomy of adult strabismus: a voxel-based morphometric analysis of magnetic resonance structural scans. Neuroimage. 2004;22(2) 986-994.

7. Farivar R, Zhou J, Huang Y, Feng L, Zhou Y, Hess RF. Two cortical deficits underlie amblyopia: a multifocal fMRI analysis. Neuroimage. Epub 2017 Sep 21.

8. Multi-ethnic Pediatric Eye Disease Study Group. Prevalence of amblyopia and strabismus in African American and Hispanic children ages 6 to 72 months the multi-ethnic pediatric eye disease study. Ophthalmology. 2008;115(7):1229-1236.

9. Bez Y, Coşkun E, Erol K, et al. Adult strabismus and social phobia: a case-controlled study. J AAPOS. 2009;13(3):249-252.

10. Mojon-Azzi SM, Kunz A, Mojon DS. Strabismus and discrimination in children: are children with strabismus invited to fewer birthday parties? Br J Ophthalmol. 2011;95(4):473-476.

11. Brown HDH, Woodall RL, Kitching RE. Using magnetic resonance imaging to assess visual deficits: a review. Ophthalmic Physiol Opt. 2016;36(3):240-265. 
12. Goodyear BG, Menon RS. Brief visual stimulation allows mapping of ocular dominance in visual cortex using fMRI. Hum Brain Mapp. 2001; 14(4):210-217.

13. Wang X, Cui D, Zheng L, Yang X, Yang H, Zeng J. Combination of blood oxygen level-dependent functional magnetic resonance imaging and visual evoked potential recordings for abnormal visual cortex in two types of amblyopia. Mol Vis. 2012;18:909-919.

14. Liang M, Xie B, Yang H, et al. Altered interhemispheric functional connectivity in patients with anisometropic and strabismic amblyopia: a resting-state fMRI study. Neuroradiology. 2017;59(5):517-524.

15. Conner IP, Odom JV, Schwartz TL, Mendola JD. Monocular activation of V1 and V2 in amblyopic adults measured with functional magnetic resonance imaging. J AAPOS. 2007;11(4):341-350.

16. Algaze A, Roberts C, Leguire L, Schmalbrock P, Rogers G. Functional magnetic resonance imaging as a tool for investigating amblyopia in the human visual cortex: a pilot study. J AAPOS. 2002;6(5):300-308.

17. Barnes GR, Hess RF, Dumoulin SO, Achtman RL, Pike GB. The cortical deficit in humans with strabismic amblyopia. J Physiol. 2001; 533(Pt 1):281-297.

18. Li C, Cheng L, Yu Q, Xie B, Wang J. Relationship of visual cortex function and visual acuity in anisometropic amblyopic children. Int $J$ Med Sci. 2012;9(1):115-120.

19. Hess RF, Thompson B, Gole G, Mullen KT. Deficient responses from the lateral geniculate nucleus in humans with amblyopia. Eur $J$ Neurosci. 2009;29(5):1064-1070.

20. Hess RF, Thompson B, Gole GA, Mullen KT. The amblyopic deficit and its relationship to geniculo-cortical processing streams. JNeurophysiol. 2010;104(1):475-483.

21. Huang X, Li SH, Zhou FQ, et al. Altered intrinsic regional brain spontaneous activity in patients with comitant strabismus: a restingstate functional MRI study. Neuropsychiatr Dis Treat. 2016;12(12): 2949-2956.

22. Tan G, Huang X, Zhang Y, et al. A functional MRI study of altered spontaneous brain activity pattern in patients with congenital comitant strabismus using amplitude of low-frequency fluctuation. Neuropsychiatr Dis Treat. 2016;12:1243-1250.

23. Li Q, Bai J, Zhang J, Gong Q, Liu L. Assessment of cortical dysfunction in patients with intermittent exotropia: an fMRI Study. PLoS One. 2016;11(8):e0160806.

24. Liu X, Yan Z, Wang T, et al. Connectivity pattern differences bilaterally in the cerebellum posterior lobe in healthy subjects after normal sleep and sleep deprivation: a resting-state functional MRI study. Neuropsychiatr Dis Treat. 2015;11:1279-1289.

25. Daw NW. Critical periods and amblyopia. Arch Ophthalmol. 1998; 116(4):502-505

26. Asper L, Crewther D, Crewther SG. Strabismic amblyopia. Part 2. Neural processing. Clin Exp Optom. 2000;83(4):200-211.

27. Vanni S, Tanskanen T, Seppä M, Uutela K, Hari R. Coinciding early activation of the human primary visual cortex and anteromedial cuneus. Proc Natl Acad Sci US A. 2001;98(5):2776-2780.

28. Liu GT, Miki A, Francis E, et al. Eye dominance in visual cortex in amblyopia using functional magnetic resonance imaging. $J$ AAPOS. 2004;8(2):184-186.

29. Goodyear BG, Nicolle DA, Humphrey GK, Menon RS. BOLD fMRI response of early visual areas to perceived contrast in human amblyopia. J Neurophysiol. 2000;84(4):1907-1913.

30. Chan ST, Tang KW, Lam KC, Chan LK, Mendola JD, Kwong KK. Neuroanatomy of adult strabismus: a voxel-based morphometric analysis of magnetic resonance structural scans. Neuroimage. 2004;22(2):986-994.

31. Li Q, Jiang Q, Guo M, Li Q, Cai C, Yin X. Grey and white matter changes in children with monocular amblyopia: voxel-based morphometry and diffusion tensor imaging study. Br J Ophthalmol. 2013;97(4): 524-529.

32. Cavanna AE, Trimble MR. The precuneus: a review of its functional anatomy and behavioural correlates. Brain. 2006;129(Pt 3):564-583.

33. Nagahama Y, Okada T, Katsumi Y, et al. Transient neural activity in the medial superior frontal gyrus and precuneus time locked with attention shift between object features. Neuroimage. 1999;10(2):193-199.
34. Lundstrom BN, Ingvar M, Petersson KM. The role of precuneus and left inferior frontal cortex during source memory episodic retrieval. Neuroimage. 2005;27(4):824-834.

35. Frings L, Wagner K, Quiske A, et al. Precuneus is involved in allocentric spatial location encoding and recognition. Exp Brain Res. 2006; 173(4):661-672.

36. Utevsky AV, Smith DV, Huettel SA. Precuneus is a functional core of the default-mode network. J Neurosci. 2014;34(3):932-940.

37. Wang T, Li Q, Guo M, et al. Abnormal functional connectivity density in children with anisometropic amblyopia at resting-state. Brain Res. 2014; $1563: 41-51$

38. Muckli L, Kiess S, Tonhausen N, Singer W, Goebel R, Sireteanu R. Cerebral correlates of impaired grating perception in individual, psychophysically assessed human amblyopes. Vision Res. 2006;46(4): 506-526.

39. Xiao JX, Xie S, Ye JT, et al. Detection of abnormal visual cortex in children with amblyopia by voxel-based morphometry. Am J Ophthalmol. 2007;143(3):489-493.

40. Liang M, Xie B, Yang H, et al. Distinct patterns of spontaneous brain activity between children and adults with anisometropic amblyopia: a resting-state fMRI study. Graefes Arch Clin Exp Ophthalmol. 2016; 254(3):569-576.

41. Ouyang J, Yang L, Huang X, et al. The atrophy of white and gray matter volume in patients with comitant strabismus: Evidence from a voxelbased morphometry study. Mol Med Rep. 2017;16(3):3276-3282.

42. Yang X, Zhang J, Lang L, Gong Q, Liu L. Assessment of cortical dysfunction in infantile esotropia using fMRI. Eur J Ophthalmol. 2014; 24(3):409-416.

43. Binkofski F, Fink GR, Geyer S, et al. Neural activity in human primary motor cortex areas $4 \mathrm{a}$ and $4 \mathrm{p}$ is modulated differentially by attention to action. J Neurophysiol. 2002;88(1):514-519.

44. Guigon E, Baraduc P, Desmurget M. Coding of movement- and forcerelated information in primate primary motor cortex: a computational approach. Eur J Neurosci. 2007;26(1):250-260.

45. Kim JA, Eliassen JC, Sanes JN. Movement quantity and frequency coding in human motor areas. J Neurophysiol. 2005;94(4):2504-2511.

46. Lin X, Ding K, Liu Y, Yan X, Song S, Jiang T. Altered spontaneous activity in anisometropic amblyopia subjects: revealed by resting-state FMRI. PLoS One. 2012;7(8):e43373.

47. Gaymard B, Ploner CJ, Rivaud S, Vermersch AI, Pierrot-Deseilligny C. Cortical control of saccades. Exp Brain Res. 1998;123(1-2):159-163.

48. Grosbras MH, Lobel E, van de Moortele PF, Lebihan D, Berthoz A. An anatomical landmark for the supplementary eye fields in human revealed with functional magnetic resonance imaging. Cereb Cortex. 1999; 9(7):705-711.

49. Vernet M, Quentin R, Chanes L, Mitsumasu A, Valero-Cabré A. Frontal eye field, where art thou? Anatomy, function, and non-invasive manipulation of frontal regions involved in eye movements and associated cognitive operations. Front Integr Neurosci. 2014;8:66.

50. Blanke O, Spinelli L, Thut G, et al. Location of the human frontal eye field as defined by electrical cortical stimulation: anatomical, functional and electrophysiological characteristics. Neuroreport. 2000; 11(9):1907-1913.

51. Fernandes HL, Stevenson IH, Phillips AN, Segraves MA, Kording KP. Saliency and saccade encoding in the frontal eye field during natural scene search. Cereb Cortex. 2014;24(12):3232-3245.

52. Tehovnik EJ, Sommer MA, Chou IH, Slocum WM, Schiller PH. Eye fields in the frontal lobes of primates. Brain Res Brain Res Rev. 2000; 32(2-3):413-448

53. Yan X, Lin X, Wang Q, et al. Dorsal visual pathway changes in patients with comitant extropia. PLoS One. 2010;5(6):e10931.

54. Tan G, Dan ZR, Zhang Y, et al. Altered brain network centrality in patients with adult comitant exotropia strabismus: a resting-state fMRI study. J Int Med Res. 2018;46(1):392-402.

55. Tang A, Chen T, Zhang J, Gong Q, Liu L. Abnormal spontaneous brain activity in patients with anisometropic amblyopia using restingstate functional magnetic resonance imaging. $J$ Pediatr Ophthalmol Strabismus. 2017;54(5):303-310. 
56. Paulin MG. The role of the cerebellum in motor control and perception. Brain Behav Evol. 1993;41(1):39-50.

57. Ohtsuka K, Enoki T. Transcranial magnetic stimulation over the posterior cerebellum during smooth pursuit eye movements in man. Brain. 1998;121(Pt 3):429-435.

58. Hayakawa Y, Nakajima T, Takagi M, Fukuhara N, Abe H. Human cerebellar activation in relation to saccadic eye movements: a functional magnetic resonance imaging study. Ophthalmologica. 2002;216(6): 399-405.

59. Kheradmand A, Zee DS. Cerebellum and ocular motor control. Front Neurol. 2011;2:53.

60. Nitschke MF, Arp T, Stavrou G. The cerebellum in the cerebrocerebellar network for the control of eye and hand movements-an fMRI study. Prog Brain Res. 2005;148:151-164.
61. Huang X, Li HJ, Zhang Y, et al. Microstructural changes of the whole brain in patients with comitant strabismus: evidence from a diffusion tensor imaging study. Neuropsychiatr Dis Treat. 2016;12:2007-2014.

62. Basso MA, Uhlrich D, Bickford ME. Cortical function: a view from the thalamus. Neuron. 2005;45(4):485-488.

63. Martin PR. Colour through the thalamus. Clin Exp Optom. 2004; 87(4-5):249-257.

64. Gupta S, Kumaran SS, Saxena R, Gudwani S, Menon V, Sharma P. BOLD fMRI and DTI in strabismic amblyopes following occlusion therapy. Int Ophthalmol. 2016;36(4):557-568.

\section{Publish your work in this journal}

Neuropsychiatric Disease and Treatment is an international, peerreviewed journal of clinical therapeutics and pharmacology focusing on concise rapid reporting of clinical or pre-clinical studies on a range of neuropsychiatric and neurological disorders. This journal is indexed on PubMed Central, the 'PsycINFO' database and CAS,

\section{Dovepress}

and is the official journal of The International Neuropsychiatric Association (INA). The manuscript management system is completely online and includes a very quick and fair peer-review system, which is all easy to use. Visit http://www.dovepress.com/testimonials.php to read real quotes from published authors.

Submit your manuscript here: http://www.dovepress.com/neuropsychiatric-disease-and-treatment-journal 